\title{
PERSISTÊNCIA NO SOLO DE INÓCULO DE Beauveria bassiana EMPREGADO PARA O CONTROLE DE Hedypathes betulinus EM PLANTIO DE Ilex paraguariensis
}

\author{
Larissa Rolim Borges ${ }^{1}$, Sônia Maria Noemberg Lazzari ${ }^{2}$, Ida Chapaval Pimentel ${ }^{3}$, \\ Lívia Cardoso Bueno de Camargo ${ }^{4}$, Lílian Mattos Kalinoski ${ }^{4}$ \\ ${ }^{1}$ Bióloga, Dr ${ }^{\mathrm{a}}$., Instituto Agronômico de Pernambuco, Recife, PE, Brasil - larissarolimborges@ gmail.com \\ ${ }^{2}$ Bióloga, Dra ., Depto. de Zoologia, UFPR, Curitiba, PR, Brasil - lazzari@ufpr.br \\ ${ }^{3}$ Eng $^{\mathrm{a}}$ Agrônoma, Dra ${ }^{\mathrm{a}}$, Depto. de Patologia Básica, UFPR, Curitiba, PR, Brasil - ida@ufpr.br \\ ${ }^{4}$ Bióloga, UFPR, Curitiba, PR, Brasil - licabuca@gmail.com; lilikalin@ gmail.com
}

Recebido para publicação: 17/08/2009 - Aceito para publicação: 29/04/2010

\begin{abstract}
Resumo
O controle da broca-da-erva-mate, Hedypathes betulinus, limita-se ao emprego de práticas silviculturais e catação manual dos insetos adultos, não sendo permitido o uso de inseticidas químicos na cultura. Esta pesquisa teve por objetivos verificar a presença natural de Beauveria bassiana no solo de plantio em monocultura de erva-mate, determinar a persistência de B. bassiana no solo após duas e três aplicações do fungo e averiguar a influência de fatores abióticos na estabilidade do fungo no solo. O estudo foi conduzido em um erval no município de Campo Alegre, Santa Catarina, Brasil, onde foram coletadas amostras de solo em duas áreas, para avaliação da presença natural de inóculos do fungo $B$. bassiana, procedendo-se à contagem, isolamento e identificação. Não foi detectada a ocorrência natural do fungo no solo em nenhuma das duas áreas. Após essa confirmação, foram realizadas pulverizações com $B$. bassiana em ambas as áreas, sendo que em uma ocorreram duas e na outra três aplicações. Após a última pulverização, realizada em fevereiro/2006, verificou-se que os inóculos do fungo persistiram por 90 dias na área com duas aplicações e 120 dias na área com três aplicações. A temperatura afetou a persistência do fungo no solo em ambas as áreas, mas a umidade relativa não mostrou influência sobre os inóculos em campo.

Palavras-chave: Broca-da-erva-mate; controle biológico; epizootia; fungo entomopatogênico.
\end{abstract}

\begin{abstract}
Persistence in soil of inoculum of Beauveria bassiana used to control Hedypathes betulinus in yerba maté plantation, Ilex paraguariensis. Control measures of Hedypathes betulinus are restricted to agricultural practices and hand picking of adult insects. No chemical control is allowed in yerba maté cultivation areas. The objectives of this research were to verify the natural occurrence of the entomopathogenic fungus Beauveria bassiana in the soil of yerba maté in monoculture; to determine the persistence of inoculums in the soil after applications of the fungus; and to evaluate the influence of abiotic factors on fungus stability in the soil. The soil samples were taken from two experimental areas in a yerba maté plantation in the county of Campo Alegre, Santa Catarina, Brazil. The samples were analyzed in order to count, isolate and identify inoculums of $B$. bassiana and other fungi. After the confirmation that $B$. bassiana was not present in the soil, a suspension of the fungus was applied, twice in one of the areas and three times in the other. After the last application in February/2006, the inoculum persisted for 90 and 120 days, in the area with two and three applications, respectively. The temperature affected the persistence of $B$. bassiana in soil; however, the relative humidity did not. Keywords: Biological control; epizooty; entomopathogenic fungus; wood borer.
\end{abstract}

\section{INTRODUÇÃO}

A erva-mate, Ilex paraguariensis St.-Hil. (Aquifoliaceae), compõe um dos sistemas florestais mais antigos e característicos da região Sul do Brasil (PENTEADO et al., 2000). Entretanto a destruição dos ambientes naturais para formação de sistemas agroflorestais favoreceu o crescimento populacional de insetos fitófagos e a redução da densidade e diversidade de inimigos naturais (DIAZ, 1997; IEDE, 1985; LEITE et al., 2006a). 
O controle biológico de insetos com a utilização de fungos entomopatogênicos, parasitoides e predadores é uma ferramenta eficaz no manejo integrado de pragas, visando a manutenção do equilíbrio ecológico em ambientes naturais e ecossistemas agrícolas (BELLOWS, 2001; HEADRICK; GROEDEN, 2001; LANZA et al., 2004).

Diversos fungos entomopatogênicos têm sido empregados no controle biológico de pragas no Brasil, em razão de sua ampla distribuição geográfica, variedade de hospedeiros e ocorrência em condições naturais, enzoótica ou epizooticamente (ALVES; LECUONA, 1998). Dentre os micoinseticidas existentes, os que apresentam conídios do fungo Beauveria bassiana (Bals.) Vuill. (Deuteromycota) como ingrediente ativo são muito utilizados e eficientes para o combate de diversas pragas (DALZOTO; UHRY, 2009).

Em erva-mate foram realizados diversos estudos visando o controle biológico de pragas com $B$. bassiana e outros fungos entomopatogênicos, com resultados promissores. Oliveira et al. (2000) testaram a patogenicidade de isolados de B. bassiana ao ácaro vermelho da erva-mate, Oligonychus yothersi (McGREGOR, 1914) (Acari: Tetranychidae), verificando que a mortalidade variou de 77 a $98 \%$ para todos os isolados. Leite et al. (2000) testaram em laboratório os fungos B. bassiana, Beauveria brongniartii (Sacc.) Petch e Metarhizium anisopliae (Metsch.) Sorok. A cepa CG 714 de B. bassiana apresentou mortalidade de 94 e 100\% em campo. Leite et al. (2006b), em testes de campo com a mesma cepa, formulada em duas concentrações de óleo emulsionado e três concentrações de conídios, verificaram que o formulado mais eficiente foi o de concentração $10^{7}$ conídios $/ \mathrm{mL}$ e $0,5 \%$ de óleo, obtendo uma infectividade de $76 \%$ em adultos de Hedypathes betulinus (KLUG, 1825) (Coleoptera: Cerambycidae). Dalla Santa (2009) testou Beauveria sp. para o controle das lagartas da erva-mate Telosia camina Schaus, 1920, e uma espécie de Hylesia Hübner, 1820 (Lepidoptera: Saturniidae), em laboratório, obtendo mortalidade superior a 90 e $49 \%$, respectivamente.

Para Alves (1998), o solo é o reservatório natural dos fungos entomopatogênicos em campo, por isso o sucesso do seu emprego em programas de controle biológico é condicionado à sua eficácia e persistência nesse ambiente. Entretanto existem fatores bióticos e abióticos que podem ser responsáveis pela sua persistência por períodos mais longos, como a temperatura, umidade relativa (GUERRA et al., 2009; RATH et al., 1992; STUDDERT; KAYA, 1990) e presença do hospedeiro específico (KESSLER et al., 2004).

Com base nessas considerações e visando ampliar as alternativas para o controle biológico de $H$. betulinus com a utilização do fungo entomopatogênico $B$. bassiana, os objetivos deste trabalho foram verificar a presença natural de B. bassiana no solo de monocultura de erva-mate, determinar a persistência de inóculos do fungo no solo após sua pulverização em campo e estabelecer possíveis interações da presença do fungo com fatores abióticos.

\section{MATERIAL E MÉTODOS}

\section{Local de coleta}

Os trabalhos de campo foram realizados em duas áreas de $5000 \mathrm{~m}^{2}$ cada, ambas localizadas em um plantio de 28 ha de monocultura de erva-mate, no município de Campo Alegre, estado de Santa Catarina (latitude de $26^{\circ} 11^{\prime} \mathrm{S}$, longitude $49^{\circ} 14^{\prime} \mathrm{W}$ ), com sete anos de implantação.

O tipo climático da região, segundo classificação de Köppen, é Cfb - mesotérmico subtropical úmido, com verões frescos, sem estação seca e com frequentes geadas severas. Temperatura média anual de $18,3{ }^{\circ} \mathrm{C}$ e precipitação média anual de aproximadamente $1500 \mathrm{~mm} / \mathrm{ano}$. Na localidade de estudo predomina a classe de solo Cambissolo álico, sendo que o horizonte "A" nessa classe, na maior parte das vezes, é proeminente e a textura é argilosa. São solos bastante suscetíveis à erosão, em relevo forte ondulado, e moderadamente susceptível quando o relevo é ondulado ou suave ondulado (EMBRAPA, 1999).

\section{Presença natural de $B$. bassiana no solo}

$\mathrm{Na}$ primeira quinzena de novembro/2005 foi realizada uma amostragem do solo nas duas áreas (A1 e A2) do plantio de erva-mate, para a verificação da ocorrência natural de B. bassiana.

Para tanto, em cada uma das duas áreas foram coletadas três amostras de solo, de aproximadamente $50 \mathrm{~g}$ cada, a uma profundidade aproximada de $0-5 \mathrm{~cm}$. As amostras foram conservadas em geladeira por aproximadamente 18 horas, até a realização das análises no Laboratório de 
Microbiologia e Biologia Molecular (LabMicro) do Departamento de Patologia Básica da Universidade Federal do Paraná (UFPR).

Cada amostra foi dividida em três subamostras, visando minimizar a heterogeneidade no solo de cada uma das áreas. Para o isolamento dos fungos, empregou-se a técnica de diluição sucessiva (CLARK, 1965), utilizando-se $10 \mathrm{~g}$ de solo úmido previamente peneirado em malha de $2 \mathrm{~mm}$ e transferido para frascos de vidro contendo $90 \mathrm{~mL}$ de solução salina $(\mathrm{NaCl}$ a $0,9 \%)$ com pérolas de vidro $(5-6 \mathrm{~mm}$ de diâmetro), agitadas por 20 minutos em agitador (100 rpm). Dessa diluição, $1 \mathrm{~mL}$ foi acrescido a $9 \mathrm{~mL}$ de água destilada esterilizada, obtendo-se a diluição de $10^{2}$. Repetiu-se esse procedimento para se obter a diluição $10^{3}$. Das diluições $10^{2}$ e $10^{3}$ retirou-se $0,1 \mathrm{~mL}$, que foi colocado em placa de Petri contendo $20 \mathrm{~mL}$ do meio batata dextrose ágar (BDA), acrescido de estreptomicina $(0,03 \mathrm{~g} / \mathrm{L})$, em três repetições por diluição. As placas foram inoculadas e incubadas em BOD a $26 \pm 1^{\circ} \mathrm{C}$.

As unidades formadoras de colônias (UFC) de B. bassiana foram isoladas em BDA, incubadas a $25 \pm 1{ }^{\circ} \mathrm{C}$ por 10 dias. Após o crescimento, os isolados foram mantidos sob refrigeração a $4{ }^{\circ} \mathrm{C}$. Para a identificação dos isolados, foram utilizados critérios macro e micromorfológicos, utilizando-se o Método de Cultura em Lâmina ou Microcultivo (KERN; BLEVINS, 1999) e chaves de identificação específicas (ALVES, 1998; KONEMAN et al., 2006). As lâminas foram fixadas em lactofenol de Amman e analisadas ao microscópio óptico, sob aumento de 100x.

\section{Seleção e aplicação do fungo $B$. bassiana em campo}

A cepa de $B$. bassiana utilizada no estudo foi isolada a partir de adultos de $H$. betulinus coletados em campo (LEITE et al., 2000) e está catalogada na coleção da Embrapa Recursos Genéticos (CENARGEM, Brasília, DF) sob o código CG 716. O fungo foi formulado em óleo emulsionável a 0,5\% na concentração de $10^{7}$ conídios/mL (LEITE et al., 2003).

As aplicações do fungo iniciaram na segunda quinzena de novembro/2005, visando o manejo do inseto $H$. betulinus nas duas áreas, sendo que na área 1 (A1) as aplicações foram feitas em novembro/2005 e fevereiro/2006, e na área 2 (A2), em novembro/2005, janeiro e fevereiro/2006. Esse cronograma foi baseado no ciclo biológico do inseto, visando a potencialização do patógeno para o controle do inseto. Assim, as aplicações ocorreram no início da emergência dos adultos (novembro) e nos períodos de maior ocorrência da praga em campo (janeiro e fevereiro) (BORGES, 2007; SOARES, 1998).

A metodologia utilizada foi a aplicação do fungo com pulverizador costal, no tronco de todas as erveiras de cada uma das áreas (aproximadamente $150 \mathrm{~mL}$ do produto/planta) (LEITE et al., 2006b). O horário de aplicação foi o vespertino (entre 17 e $18 \mathrm{~h}$ ), pois os efeitos da radiação ultravioleta (UV) reduzem a eficiência do fungo no campo (BRAGA et al., 2001).

\section{Persistência de inóculos $B$. bassiana no solo}

As coletas para verificação da persistência do fungo no solo, nas duas áreas, foram realizadas mensalmente entre os meses de dezembro/2005 e outubro/2006, totalizando 11 coletas. A metodologia de coleta do solo nas áreas foi a mesma descrita anteriormente.

\section{Avaliação de possíveis interações de $B$. bassiana com fatores abióticos}

Os dados obtidos a partir do isolamento das colônias de $B$. bassiana foram submetidos a análise estatística pelo teste de Tukey, ao nível de 5\%, para verificar se a diferença observada entre as áreas foi significativa. Realizou-se a análise de correlação de Pearson para comparar o número de colônias do fungo com a temperatura média e a umidade relativa. Os dados meteorológicos foram obtidos da Empresa Modo Battistella Indústria e Comércio S.A., na localidade de Rio Negrinho, SC.

\section{RESULTADOS E DISCUSSÃO}

\section{Ocorrência natural de $B$. bassiana no solo}

Não foi constatada a ocorrência de $B$. bassiana em amostras de solo em nenhuma das duas áreas. Corroborando esse resultado, a presença natural desse fungo não foi verificada em solo ou rizosfera de ambientes agrícolas e florestais, como o girassol (CAVALCANTI et al., 2003), cana-de-açúcar (SANTOS et al., 1989), tomate (SILVA et al., 1990), soja e algodão (CARVALHO, 2008), goiaba (SILVÉRIO, 2007), uva-do-japão (PRADE et al., 2006) e citros (PRADE et al., 2007). Em levantamentos da diversidade fúngica realizados em diversos biomas brasileiros, como a Caatinga (COSTA et al., 2006; SIMÕES; TAUK-TORNISIELO, 2006), Cerrado (CARVALHO, 2008) e Mata Atlântica (GARLIPP, 1995; TAUK-TORNISIELO et al., 2005), esse patógeno também não foi encontrado. 
Entretanto, segundo Alves (1998), Boucias; Pendland (1998) e Quesada-Moraga et al. (2007), esse fungo possui ampla distribuição geográfica e faz parte da flora microbiana do solo, podendo ser isolado, principalmente, em ambientes naturais. Em erva-mate foram registradas epizootias naturais desse fungo em $H$. betulinus por Soares et al. (1995) e Silva et al. (1998), no município de Ivaí, PR. Soares; Iede (1997) inferiram que B. bassiana pode ocorrer naturalmente em ervais estremes, indicando sua adaptação ao ecossistema modificado.

\section{Persistência de inóculos $B$. bassiana no solo}

Após a última aplicação de B. bassiana no tronco das árvores nas duas áreas, em fevereiro/2006, observou-se que a persistência do fungo no solo foi de aproximadamente 90 dias na área 1 (A1) e 120 dias na área 2 (A2), ambos na concentração $10^{3}$ conídios/mL (Figura 1). No teste de comparação de médias com o número de colônias nas duas áreas, ao longo de todo o período experimental, verificou-se que estas não diferiram entre si (Tabela 1).

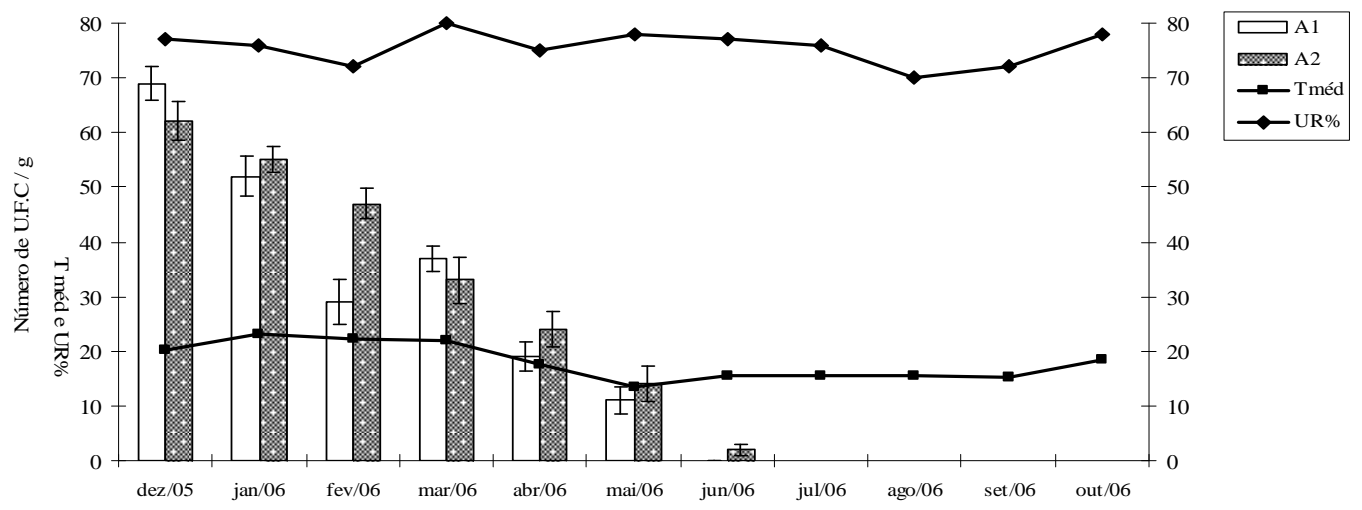

Figura 1. Número de unidades formadoras de colônias de B. bassiana (UFC/g) x $10^{3}$ isoladas do solo em áreas de povoamento puro de erva-mate, com relação a temperatura média e umidade relativa, no município de Campo Alegre, SC, Brasil, de dezembro/2005 a outubro/2006. Áreas: A1 com duas aplicações do formulado fúngico; A2 - com três aplicações.

Figure 1. Number of colony forming unities of $B$. bassiana $(\mathrm{CFU} / \mathrm{g}) \times 10^{3}$ isolated from soil of areas of cultivation of yerba mate, related to mean temperature and relative humidity in the county of Campo Alegre, SC, Brazil, from December/2005 to October/2006. Areas: A1 - two applications of the fungus formulation; and A2 - three applications of the fungus.

Tabela 1. Número total e médio de unidades formadoras de colônias de $B$. bassiana (UFC/g) x $10^{3}$ isoladas do solo em áreas de povoamento puro de erva-mate nas áreas A1 (com duas aplicações do formulado fúngico) e A2 (com três aplicações), no município de Campo Alegre, SC, Brasil, de dezembro/2005 a outubro/2006.

Table 1. Total and mean number of colony forming unities of B. bassiana $(\mathrm{CFU} / \mathrm{g}) \times 10^{3}$ isolated from soil of areas of cultivation of yerba mate in areas A1 (two applications of the fungus formulation) and A2 (three applications of the fungus), in the county of Campo Alegre, SC, Brazil, from December/2005 to October/2006.

\begin{tabular}{lcc}
\hline Áreas & Número total de UFC & Número médio de UFC \\
\hline $\mathrm{A} 1$ & $217 \mathrm{a}$ & $33,16 \pm 21,48$ \\
$\mathrm{~A} 2$ & $237 \mathrm{a}$ & $33,85 \pm 22,05$ \\
\hline
\end{tabular}

*Notas seguidas de letras diferentes diferem entre si pelo teste de Tukey ao nível de 5\%.

A concentração de conídios recuperada do solo, nas duas áreas, provavelmente pode atuar como fonte de inóculo do fungo, causando infecção no hospedeiro. Essa hipótese é corroborada por Alves; Lecuona (1998), que relataram que $10^{3}$ conídios/inseto de $B$. bassiana são capazes de provocar 
mortalidade em Anthomonus grandis Boheman, 1843 (Coleoptera: Curculionidae). Além disso, Oliveira et al. (2008) verificaram alterações significativas na biologia reprodutiva de Diatraea saccharalis (Fabricius, 1794) (Lepidoptera: Crambidae) infectados com B. bassiana a partir da concentração $10^{3}$ conídios/mL, resultando em queda no ritmo de crescimento da população e originando gerações com menos indivíduos.

A partir dos meses de junho e julho/2006, a ausência de inóculo no solo pode ter sido influenciada pela redução dos níveis populacionais de adultos de $H$. betulinus em campo, relatado por Borges (2007). Walstad et al., citados por Guerra et al. (2009), consideram improvável o crescimento de $B$. bassiana em abundância como sapróbio no solo, uma vez que o desenvolvimento do seu ciclo biológico também depende de uma fase parasitária no hospedeiro. Assim, a relação estreita entre patógeno-hospedeiro leva a um controle mútuo, expresso na flutuação da população do hospedeiro (GAUGLER et al., 1989; KELLER; ZIMMERMANN, 1989; STOREY et al., 1989), podendo, contudo, o patógeno continuar presente em baixos níveis no solo (KELLER et al., 2003).

Avaliação de possíveis interações de $B$. bassiana com fatores abióticos

A correlação entre o número de colônias de B. bassiana recuperadas no solo com fatores abióticos indica que a temperatura média (que variou de 13,5 a $23,2{ }^{\circ} \mathrm{C}$ ) influenciou na persistência do fungo, não sendo afetada, porém, pela umidade relativa (Tabela 2).

Para Quintela (1986), a temperatura é um dos fatores que mais compromete a persistência de $B$. bassiana em condições de campo. Alves; Lecuona (1998) corroboram essa informação e afirmam que esse fungo apresenta uma faixa ótima de desenvolvimento na temperatura entre 23 e $26{ }^{\circ} \mathrm{C}$, podendo suportar temperaturas de até $45^{\circ} \mathrm{C}$. Lingg; Donaldson (1981) avaliaram a sobrevivência de B. bassiana no solo e verificaram que ela foi primariamente dependente da temperatura e do conteúdo da água, pois os esporos mantidos em altas temperaturas não foram recuperados em nenhuma das umidades testadas.

No presente estudo, a umidade relativa não se apresentou como fator primordial para a persistência do fungo no solo (Tabela 2), porém esse fato pode estar relacionado à pequena variação da umidade ao longo do período experimental, com média de 75,5\% (variando entre 71 e $80 \%$ ). Para Alves; Lecuona (1998), a umidade desempenha um importante papel para o patógeno, sendo essencial para as fases de disseminação, germinação e penetração, e limitante para a reprodução de alguns patógenos, sendo considerada tão importante quanto à temperatura. Entretanto, para esses autores, a umidade relativa que está presente sobre os insetos ou nos locais onde eles vivem (nível microclimático) pode ser mais importante para a ocorrência da doença quando comparada com a umidade relativa atmosférica (nível macroclimático).

Tabela 2. Correlação do número de colônias de B. bassiana com os fatores abióticos temperatura média e umidade relativa nas áreas A1 (duas aplicações do formulado fúngico) e A2 (três aplicações), no município de Campo Alegre, SC, Brasil, de dezembro/2005 a outubro/2006.

Table 2. Correlation between the number of colonies of $B$. bassiana with the abiotic factors mean temperature and relative humidity for areas A1 (two applications of the fungus formulation) and A2 (three applications of the fungus), in the county of Campo Alegre, SC, Brazil, from December/2005 to October/2006.

\begin{tabular}{lcc}
\hline Fatores climáticos & $\mathbf{A 1}$ & $\mathbf{A 2}$ \\
\hline Temperatura média $\left({ }^{\circ} \mathrm{C}\right)$ & $\mathrm{r}=0,70^{*}$ & $\mathrm{r}=0,79^{*}$ \\
Umidade relativa $(\%)$ & $\mathrm{r}=0,03$ & $\mathrm{r}=-0,27$ \\
\hline
\end{tabular}

* Valor de $\mathrm{r}$ maior que 0,7 indica forte correlação.

\section{CONCLUSÕES}

- Não se constatou a ocorrência natural de B. bassiana em solo de erval em Campo Alegre, SC.

- Após a última pulverização do formulado fúngico, no mês de fevereiro/2006, foram detectados inóculos de B. bassiana que persistiram por 90 dias na área com duas aplicações e 120 dias na área com três aplicações.

- A persistência do inóculo em ambas as áreas apresentou correlação com a temperatura média. 


\section{AGRADECIMENTOS}

Ao CNPq, pela bolsa de doutorado concedida à primeira autora e de pesquisa para a segunda; aos pesquisadores do laboratório de Entomologia da Embrapa Florestas; à Dra. Maria Silvia P. Leite, pela produção do fungo; aos professores Juarez Gabardo e Júlio Valaski, pelas análises estatísticas; e à Empresa Modo Battistella Indústria e Comércio S.A., pela disponibilização dos dados meteorológicos.

\section{REFERÊNCIAS}

ALVES, S. B. Controle microbiano de insetos. Piracicaba, SP: FEALQ, 1998. 1163 p.

ALVES, S. B.; LECUONA, R. E. Epizootiologia aplicada ao controle microbiano In: ALVES, S. B. Controle microbiano de insetos. 2. ed. Piracicaba, SP: FEALQ, 1998, p. 97-170.

BELLOWS, T. S. Restoring population balance through natural enemy introductions. Biological Control, v. 21, p. 199-205, 2001.

BORGES, L. R. Eficiência de Beauveria bassiana (Bals.) Vuill. (Deuteromycota) para o controle de Hedypathes betulinus (Klug) (Coleoptera: Cerambycidae) em erva-mate, Ilex paraguariensis St.-Hil. (Aquifoliaceae). 101 f. Tese (Doutorado em Entomologia) - Universidade Federal do Paraná, Curitiba, 2007.

BOUCIAS, D. G.; PENDLAND, J. C. Principles of insect pathology. Boston: Kluwer, 1998. 548 p.

BRAGA, G. U. L.; FLINT, S. D.; MESSIAS, C. L.; ANDERSON, A. J.; ROBERTS, D. W. Effects of UV-B irradiance on conidia and germinants of the entomopathogenic hyphomycete Metarhizium anisopliae: a study of reciprocity and recovery. Photochemistry and Photobiology, v. 72, p.140-146, 2001.

CARVALHO, V. G. Comunidades de fungos em solo do cerrado sob vegetação nativa e sob cultivo de soja e algodão. 62 f. Dissertação (Mestrado em Microbiologia Agrícola) - Universidade Federal de Lavras, Lavras, 2008.

CAVAlCANTI, M. A. Q.; MOTTA, C. M. S.; LIMA, D. M.; LARANJEIRA, D.; FERNANDES, M. J. S. Identification and characterization of filamentous fungi isolates from the sunflower (Helianthus annus L.) rhizosphere according to their capacity to hydrolyse inulin. Brazilian Journal of Microbiology, v. 34, p. 273-280, 2003.

CLARK, F. E. Agar-plate method for total microbial count. In: BLACK, C. A; EVANS, D. D.; WHITE, J. L.; ENSMINGER, L. E.; CLARK, F. E.; DINAVER, R. C. (eds.). Methods of soil analysis, Part 2. Chemical and microbiological properties. New York: Madson Inc., 1965. p. 1460-1466.

COSTA, I. P. M. W.; CAVALCANTI, M. A. Q.; FERNANDES, M. J. S.; LIMA, M. M. Hypomycetes from soil of an area affected by copper mining activities in the state of Bahia, Brazil. Brazilian Journal of Microbiology, v. 37, p. 290-295, 2006.

DALLA SANTA, H. S.; SOUSA, N. J.; PITTNER, E.; DALLA SANTA, O. R.; SOCCOL, C. R. Controle biológico em pragas de Ilex paraguariensis (A. St.-Hil.) com fungo Beauveria sp. Revista Floresta, v. 39, p. 67-76, 2009.

DALZOTO, P. R.; UHRY, K. F. Controle biológico de pragas no Brasil por meio de Beauveria bassiana (Bals.) Vuill. Reunião Anual do Instituto Biológico, v. 71, p. 37-41, 2009.

DIAZ, C. I. F. Perspectivas del manejo integrado de plagas em yerba mate. In: I Congresso SulAmericano da Erva-Mate e II Reunião Técnica do Cone Sul sobre a Cultura da Erva-mate. 1997, Curitiba, Ed. dos Organizadores. Anais... Curitiba: 1997. p. 371-390.

EMBRAPA. Empresa Brasileira de Pesquisa Agropecuária. Sistema Brasileiro de Classificação de Solos. SPDI - Embrapa Solos. Rio de Janeiro. 1999. 412 p. 
GARLIPP, A. B. Isolamento e identificação de fungos filamentosos do solo do Banhado Grande, na Estação Ecológica de Jureia-Martins, SP. 94 f. Dissertação (Mestrado em Microbiologia Aplicada) Instituto de Biociências, UNESP, Jaboticabal, 1995.

GAUGLER, R.; COSTA, S. D.; LASHOMB, J. Stability and efficacy of Beauveria bassiana soil inoculations. Environ. Entomol., v. 18, p. 412-417, 1989.

GUERRA, D. M. S.; PIRES, A. P. D.; LIMA, E. A. L. A. Persistência de Metarhizium anisopliae no solo sob diferentes condições de temperatura e umidade. Revista Caatinga, v. 22, n. 1, p. 50-54, 2009.

HEADRICK, D. H.; GROEDEN, R. D. Biological control as a tool for ecosystem management. Biological Control, v. 21, p. 249-257, 2001.

IEDE, E. T. Considerações sobre a entomofauna da erva-mate (Ilex paraguariensis St.-Hil.). In: Seminário sobre atualidades e perspectivas florestais - Silvicultura da erva-mate (Ilex paraguariensis St.Hil.). 1985, EMBRAPA/CNPF, Colombo. Anais... Colombo: 1985. 146 p.

KELLER, S.; ZIMMERMANN, G. Mycopathogens of soil insects. In: WILDING, N.; COLLINS, N. M.; HAMMOND, P. M.; WEBBER, J. F. (eds.). Insect-Fungus Interactions. London: Academic Press, 1989. p. 239-270.

KELLER, S.; KESSLER, P.; SCHWEIZER, C. Distribution of insect pathogenic soil fungi in Switzerland with special reference to Beauveria brongniartii and Metarhizium anisopliae. Biocontrol, v. 48, p. 307319. 2003.

KERN, M. E.; BLEVINS, K. S. Micologia médica. 2. ed. São Paulo: Editora Premier, 1999. 256 p.

KESSLER, P.; ENKERLI, J.; SCHWEIZER, C.; KELLER, S. Survival of Beauveria brongniartii in the soil after application as biocontrol agent against the European cockchafer Melolontha melolontha. Biocontrol, v. 59, p. 563-581, 2004.

KONEMAN, E. W.; ALLEN, S. D.; JANDA, W. M.; SCHRECKENBERGER, P. C.; WINN JR., W. C. Diagnóstico microbiológico. São Paulo: Editora MEDSI, 2006. 221 p.

LANZA, L. M.; MONTEIRO, A. C.; MALHEIROS, E. B. População de Metarhizium anisopliae em diferentes tipos e graus de compactação do solo. Ciência Rural, v. 34, p. 57-62, 2004.

LEITE, M. S. P.; SOARES, C. M. S.; IEDE, E.T.; PENTEADO, S. R. C.; CASTELLANO, C. Seleção de linhagens de fungos entomopatogênicos para o controle de Hedypathes betulinus (Klug, 1825) (Coleoptera: Cerambycidae) em laboratório e eficiência da linhagem selecionada em campo. In: II Congresso Sul-Americano da Erva-Mate e III Reunião Técnica da Erva-mate. 2000, Encantado, RS, Ed. dos Organizadores. Anais... Encantado, 2000. p. 314-317.

LEITE, M. S. P.; PENTEADO, S. R. C.; OLIVEIRA, S. Eficiência do fungo Beauveria bassiana (Bals.) Vuill. no controle de Hedypathes betulinus (Klug, 1825) (Coleoptera: Cerambycidae) em campo. In: III Congresso Sul-Americano de Erva-Mate. 2003, Chapecó, Santa Catarina. Anais... Chapecó, 2003. CdRom.

LEITE, M. S. P.; IEDE, E. T.; PENTEADO, S. R. C. Manejo integrado de pragas em erva-mate para o controle da broca-da-erva-mate, com ênfase ao uso de entomopatógenos. 2006a. In: IV Congreso Sudamericano de la Yerba Mate, Misiones. Anais... Misiones: 2006a, Cd-Rom.

LEITE, M. S. P.; IEDE, E. T.; PENTEADO, S. R. C.; ZALESKI, S. R. M.; CAMARGO, J. M. M.; RIBEIRO, R. D. Eficiência de Beauveria bassiana (Bals.) Vuill. formulado em óleo no controle de Hedypathes betulinus (Klug) (Coleoptera: Cerambycidae) em campo. 2006b. In: IV Congreso Sudamericano de la Yerba Mate, Misiones. Anais... Misiones: 2006b. p. 269-272.

LINGG, A. J.; DONALDSON, D. M. Biotic and abiotic factors affecting stability of Beauveria bassiana conidia in soil. Journal of Invertebrate Pathology, v. 39, p. 191-200. 1981. 
OLIVEIRA, M. A. P.; MARQUES, E. N. J.; WANDERLEY-TEIXEIRA, V.; BARROS, R. Efeito de Beauveria bassiana (Bals.) Vuill. e Metarhizium anisopliae (Metsch.) Sorok. sobre características biológicas de Diatraea saccharalis F. (Lepidoptera: Crambidae). Acta Sci. Biol. Sci., v. 30, n. 2, p. 220$224,2008$.

OLIVEIRA, R. C.; ALVES, L. F. A.; NEVES, P. M. O. J. Patogenicidade do fungo Beauveria bassiana ao ácaro vermelho da erva-mate. In: II Congresso Sul-Americano da Erva-Mate e III Reunião Técnica da Erva-Mate. 2000, Encantado, RS, Ed. dos Organizadores. Anais... Encantado, 2000. p. 318-321.

PENTEADO, S. R. C.; IEDE, E. T.; LEITE, M. S. P. Pragas da erva-mate: perspectivas de controle. In: II Congresso Sul-Americano da Erva-Mate e III Reunião Técnica da Erva-Mate. 2000, Encantado, RS, Ed. dos Organizadores. Anais... Encantado, 2000. p. 27-38.

PRADE, C. A.; MATSUMURA, A. T. S.; GUERRERO, R. T.; PORTO, M. L. Diversidade de fungos filamentosos e microscópicos do solo em uma plantação de Hovenia dulcis Thumb. Biociências, v. 14, n. 2, p. 101-106, 2006.

PRADE, C. A.; MATSUMURA, A. T. S.; OTT, A. P.; PORTO, M. L. Diversidade de fungos do solo em sistemas agroflorestais de citrus com diferentes tipos de manejo no município de Roca Sales, Rio Grande do Sul, Brasil. Biociências, v. 15, p. 73-81, 2007.

QUESADA-MORAGA, E.; NAVAS-CORTÉS, J. A.; MARANHÃO, E. A. A.; ORTIZ-URQUIZA, O. C.; SANTIAGO-ALVAREZ, C. Factors affecting the occurrence and distribution of entomopathogenic fungi in natural and cultivated soils. Mycological Research, v. 111, p. 947-966, 2007.

QUINTELA, E. D. Estabilidade de Beauveria bassiana (Bals.) Vuill. (Hyphomycetes) no solo e sua patogenicidade ao Chalcodermus aeneus Boheman (Col: Curculionidae), praga do caupi. $101 \mathrm{f}$. Dissertação (Mestrado em Entomologia) - ESALQ/USP, Piracicaba, 1986.

RATH, A. C.; KOEN, T. B.; YIP, H. Y. The influence of abiotic factors on the distribution and abundance of Metarhizium anisopliae in Tasmanian pastures soils. Mycological Research, v. 96, p. 378384, 1992.

SANTOS, A. C.; CAVAlCANTI, M. A. Q.; FERNANDES, M. J. S. Fungos isolados da rizosfera da cana-de-açúcar da Zona da Mata de Pernambuco. Revista Brasileira de Botânica, v. 12, 23-29, 1989.

SILVA, C. F.; PEDROSA-MACEDO, J. H.; SOUSA, N. J. Infecção de Thelosia camina Schaus, 1920 (Lepidoptera, Eupterotidae) "lagarta da erva-mate" por Beauveria spp. In: Congresso Brasileiro de Entomologia, 1998, Rio de Janeiro, RJ, UFRRJ. Anais... Rio de Janeiro, 1998. p. 769.

SILVA, M. I. L.; CAVALCANTI, M. A. Q.; LIMA, D. M. M. Fungos da rizosfera de sementeira de tomate. Fitopatologia Brasileira, v. 15, 323-326, 1990.

SILVÉRIO, M. L. Fungos filamentosos isolados da rizosfera de plantas nativas da caatinga e cultivos de goiabeiras (Psidium guajava L.) sadias e infestadas por nematoides. 47 f. Dissertação (Mestrado em Biologia de Fungos) - Universidade Federal de Pernambuco, Recife, 2007.

SIMÕES, M. L. G.; TAUK-TORNISIELO, S. M. Optimization of xylanase biosynthesis by Aspergillus japonicus isolated from a "Caatinga" area in the Brazilian state of Bahia. African Journal of Biotechnology, v. 5, p. 1135-1141, 2006.

SOARES, C. M. S. Flutuação populacional, aspectos comportamentais e levantamento de inimigos naturais de Hedypathes betulinus (Klug, 1825) (Coleoptera: Cerambycidae) em um povoamento puro de erva-mate (Ilex paraguariensis St.-Hil.). 73 f. Tese (Doutorado em Entomologia) Universidade Federal do Paraná, Curitiba, 1998.

SOARES, C. M. S.; IEDE, E. T.; SANTOS, H. R. Ocorrência natural dos fungos entomopatogênicos Beauveria bassiana e Metarhizium anisopliae sobre Hedypathes betulinus (Coleoptera: Cerambycidae). In: V Siconbiol - Simpósio de Controle Biológico, 1995, Foz do Iguaçu, PR. Ed. Embrapa. Anais... Foz do Iguaçu, 1995. p. 81. 
SOARES, C. M. S.; IEDE, E. T. Perspectivas para o controle da broca-da-erva-mate Hedypathes betulinus (Klug, 1825) Coleoptera: Cerambycidae. In: I Congresso Sul-Americano da Erva-Mate e II Reunião Técnica do Cone Sul sobre a Cultura da Erva-Mate. Curitiba, Ed. dos Organizadores. Anais... Curitiba, 1997. p. 391-400.

STOREY, G. K.; GARDNER, W. A.; TOLLNER, E. W. Penetration and persistence of commercially formulated Beauveria bassiana conidia in soil of two tillage systems. Environmental Entomology, n. 18 , p. 835-839. 1989.

STUDDERT, J. P.; KAYA, H. K. Water potential, temperature and soil type on the action of Beauveria bassiana soil colonies. Journal of Invertebrate Pathology, v. 56, p. 380-383, 1990.

TAUK-TORNISIELO, S. M.; GARLIPP, A. B.; RUEGGER, M.; ATTILI, D. S.; MALAGUTTI, E. Soilborne filamentous fungi in Brazil. Brazilian Journal of Microbiology, v. 45, p. 72-82, 2005. 
FLORESTA, Curitiba, PR, v. 41, n. 1, p. 135-144, jan./mar. 2011

Borges, L. R. et al. 